\title{
An update of the constraints on the phenomenological MSSM from the new LHC Higgs results
}

\author{
Alexandre Arbey ${ }^{\mathrm{a}, \mathrm{b}, \mathrm{c}}$, Marco Battaglia ${ }^{\mathrm{d}, \mathrm{c}}$, Abdelhak Djouadi ${ }^{\mathrm{e}}$, \\ Farvah Mahmoudi ${ }^{\mathrm{f}, \mathrm{c}}$ \\ ${ }^{a}$ Université de Lyon, France; Université Lyon 1, F-69622 Villeurbanne Cedex, France \\ ${ }^{b}$ Centre de Recherche Astrophysique de Lyon, Observatoire de Lyon, Saint-Genis Laval \\ Cedex, F-69561, France; CNRS, UMR 5574; Ecole Normale Supérieure de Lyon, Lyon, \\ France \\ ${ }^{c}$ CERN, CH-1211 Geneva 23, Switzerland \\ ${ }^{d}$ Santa Cruz Institute of Particle Physics, University of California, Santa Cruz, CA \\ 95064, USA \\ ${ }^{e}$ Laboratoire de Physique Théorique, Université Paris XI and CNRS, F-91405 Orsay, \\ France \\ ${ }^{f}$ Clermont Université, Université Blaise Pascal, CNRS/IN2P3, LPC, BP 10448, F-63000 \\ Clermont-Ferrand, France
}

\begin{abstract}
Updated results on the search of Higgs bosons at the LHC with up to $17 \mathrm{fb}^{-1}$ of data have just been presented by the ATLAS and CMS collaborations. New constraints are provided by the LHCb and XENON experiments with the observation of the rare decay $B_{s} \rightarrow \mu^{+} \mu^{-}$and new limits on dark matter direct detection. In this paper, we update and extend the results on the implications of these data on the phenomenological Minimal Supersymmetric extension of the Standard Model (pMSSM) by using high statistics, flat scans of its 19 parameters. The new LHC data on $b \bar{b}$ and $\tau \tau$ decays of the lightest Higgs state and the new CMS limits from the $\tau \tau$ searches for the heavier Higgs states set stronger constraints on the pMSSM parameter space.
\end{abstract}

Keywords:

\section{Introduction}

The first results on the mass and decay rates of the Higgs-like particle observed by ATLAS and CMS at the LHC [1, 2] already imply some significant bounds on the parameters of the Minimal Supersymmetric extension of the 
Standard Model (MSSM), once we interpret the newly discovered particle as the lightest $h$ state in this theory. New results for the properties of this particle have just been presented by the ATLAS and CMS experiments with up to $17 \mathrm{fb}^{-1}$ of 7 and $8 \mathrm{TeV}$ data [3, 4, 5, 6, 7, , 8, 9, 10, 11]. New results on the search for tau lepton pairs at high invariant masses reported by the CMS collaboration [12] set tighter constraints on heavier Higgs particles. On another front the $\mathrm{LHCb}$ collaboration has obtained the first observation of the $B_{s}^{0} \rightarrow \mu^{+} \mu^{-}$rare decay and reported a first determination of its decay branching fraction [13]. Constraints on weakly interacting dark matter particles have also been significantly improved since the Higgs-like particle discovery with the updated results on the direct search by the XENON collaboration [14]. All these new data have a drastic impact not only on the SM but also on theories of supersymmetry and in particular the MSSM.

In Ref. [15] we presented a detailed analysis of the implications of the observation of a Higgs-like particle and the first determination of its properties. There, we refined a previous study [16] of the implications of the value $M_{h} \approx 126 \mathrm{GeV}$ for both the constrained and unconstrained versions of the MSSM and analysed the impact of the first data for the newly observed particle. By reviewing the different regimes of the MSSM, we concluded that two of those exhibited the best agreement with its properties: i) the decoupling regime in which the $h$ boson has SM-like properties with the $H, H^{ \pm}$and $A$ bosons being heavy and decoupled from the gauge bosons and ii) a regime where light SUSY particles such as tau-sleptons, charginos and third generation scalar quarks affected the $h$ boson rates, in particular in the $h \rightarrow \gamma \gamma$ channel.

The study presented in this paper updates that work with a focus on the regions of pMSSM parameters allowed, and favoured, by the latest LHC Higgs data and other results. We evaluate the constraints obtained in the framework of the phenomenological MSSM (pMSSM), with the neutralino as the lightest SUSY particle (LSP), with 19 free parameters using flat parameter scans. Our analysis tests the compatibility of a large sample of generated pMSSM points, fulfilling the constraints from other MSSM searches at LEP and the LHC, flavour physics data and dark matter searches and the direct searches for supersymmetric particles in channels with missing transverse energy at the LHC. The qualitative results of our previous study [15] stay the same, and are even strengthened; there are quantitative changes which make this update interesting. While the statistical accuracy of the LHC results is still limited and the data have not settled, we expect a steady improvement 
with the analysis of the full statistics collected in the $8 \mathrm{TeV}$ run and then with higher energy LHC operation from 2014. The present study provides conclusions which are already a useful guidance for the current SUSY searches at the LHC and it defines a template for further analyses, once results with better precision will be available.

The essential elements of the pMSSM scans with the various constraints and the relevant ranges for the variation of its parameters have been already presented in Ref. [15]. The tools used to perform our analysis given in Ref. [17]. Here we proceed to the presentation of our updated analysis and its results in the next section. Section 3 has a short conclusion.

\section{Analysis and Results}

The analysis is based on the latest results for the mass of the new Higgslike particle and its signal strengths in the individual channels. We use a weighted average of the results reported by the ATLAS and CMS collaborations at the LHC and also CDF and D0 at the Tevatron [18] with their estimated statistical uncertainties, as summarised in Table 1. In the following, we use the notation $R_{X X}$ to indicate the ratio of the $h$ branching fraction to the final state $X X, \mathrm{BR}(h \rightarrow X X)$, to its $\mathrm{SM}$ value. Then, we compute the so-called "signal strengths", i.e. the ratios of the products of production cross sections times decay branching fractions for the pMSSM points to their SM values, which we denote with $\mu_{X X}$ for a given $h \rightarrow X X$ channel, $\mu_{X X}=\sigma(h) / \sigma\left(H_{\mathrm{SM}}\right) \times R_{X X}$, where $\sigma$ is the relevant production cross section.

The signal strengths corresponding to each accepted pMSSM point are compared to their experimental values. Both ATLAS and CMS have provided updates for the $Z Z, W W, b \bar{b}$ and $\tau \tau$ channels with the full $7 \mathrm{TeV}$ statistics of $4.7 \mathrm{fb}^{-1}$ and $\simeq 13 \mathrm{fb}^{-1}$ of the $8 \mathrm{TeV}$ data. ATLAS has also updated the result for the $\gamma \gamma$ channel based on $4.8+13 \mathrm{fb}^{-1}$. These updates result in improved determinations of the signal strengths in the $\gamma \gamma, W W$ and $Z Z$ channel and data from both LHC experiments in the important $b \bar{b}$ and $\tau \tau$ channels. We also include the combined Tevatron result for the $b \bar{b}$ channel. While these results are compatible with the SM expectations, or the MSSM in the decoupling regime with heavy SUSY particles, within their present accuracy, the situation with the data for most of the channels does not appear to have settled. After the ATLAS update, the results for the $\gamma \gamma$ channel hint

more significantly to a possible enhancement of its rate, but a confirmation 
from CMS with the larger $8 \mathrm{TeV}$ data set would be comforting. Results on the $W W$ and $Z Z$ yields are aligned at values which are consistently about $1 \sigma$ above the SM expectation for ATLAS and $1 \sigma$ below it for CMS. The important, but experimentally difficult, $b \bar{b}$ channel still requires more data and a careful control of the SM backgrounds. At present, the spread of the experimental results by ATLAS and CMS, covers the range of values predicted by the MSSM, as we highlight in Figure 1 .

\begin{tabular}{|c|c|c|}
\hline Parameter & Value & Experiment \\
\hline \hline$M_{h}$ & $126 \pm 2 \mathrm{GeV}$ & ATLAS [1] + CMS [2] \\
$\mu_{\gamma \gamma}$ & $1.71 \pm 0.26$ & ATLAS [3] + CMS [5] \\
$\mu_{Z Z}$ & $0.97 \pm 0.26$ & ATLAS [4] + CMS [6] \\
$\mu_{W W}$ & $0.85 \pm 0.23$ & ATLAS [7] + CMS [6] \\
\hline$\mu_{b \bar{b}}$ & $1.28 \pm 0.45$ & ATLAS [8] + CMS [9] + CDF + D0) [18] \\
$\mu_{\tau \tau}$ & $0.71 \pm 0.42$ & ATLAS [10] + CMS [11] \\
\hline$D_{\gamma \gamma}$ & $1.88 \pm 0.46$ & \\
$D_{\tau \tau}$ & $0.79 \pm 0.49$ & \\
\hline
\end{tabular}

Table 1: Input values for the Higgs mass and rates used for the study.

We perform an analysis of the compatibility of the MSSM with these results, based on our set of $2.0 \times 10^{8}$ pMSSM points, with the assumption that the observed particle is the lightest Higgs boson of the MSSM, $h$, and we comment on the possible identification of the particle with the $H$ state later in this section. We first select points fulfilling all the constraints discussed in Ref. [15] but including the new measurements. In particular, for the decay $B_{s} \rightarrow \mu^{+} \mu^{-}$, the $\mathrm{LHCb}$ collaboration reported a branching ratio of $\mathrm{BR}\left(B_{s}^{0} \rightarrow \mu^{+} \mu^{-}\right)=\left(3.2_{-1.2}^{+1.5}\right) \times 10^{-9}$ with $2.2 \mathrm{fb}^{-1}$ of data [13]. We derive the constraint at $90 \%$ C.L. after accounting for theoretical uncertainties, estimated to be at the $11 \%$ level [19]. For the $b \rightarrow s \gamma$ decay branching ratio, the new world average value of $(3.43 \pm 0.22) \times 10^{-4}[20$ ] is now closer to the SM prediction leading to more severe constraints. The dark matter constraints are also updated for the direct detection limits by including the new result on the spin-independent $\chi$-nucleon scattering cross section from 225 live days of XENON-100 data [14]. We note that for the Higgs decay branching fractions, we use the latest version of HDECAY (5.0) [21] which includes, among the new features, a more refined treatment of the SUSY vertex corrections. 

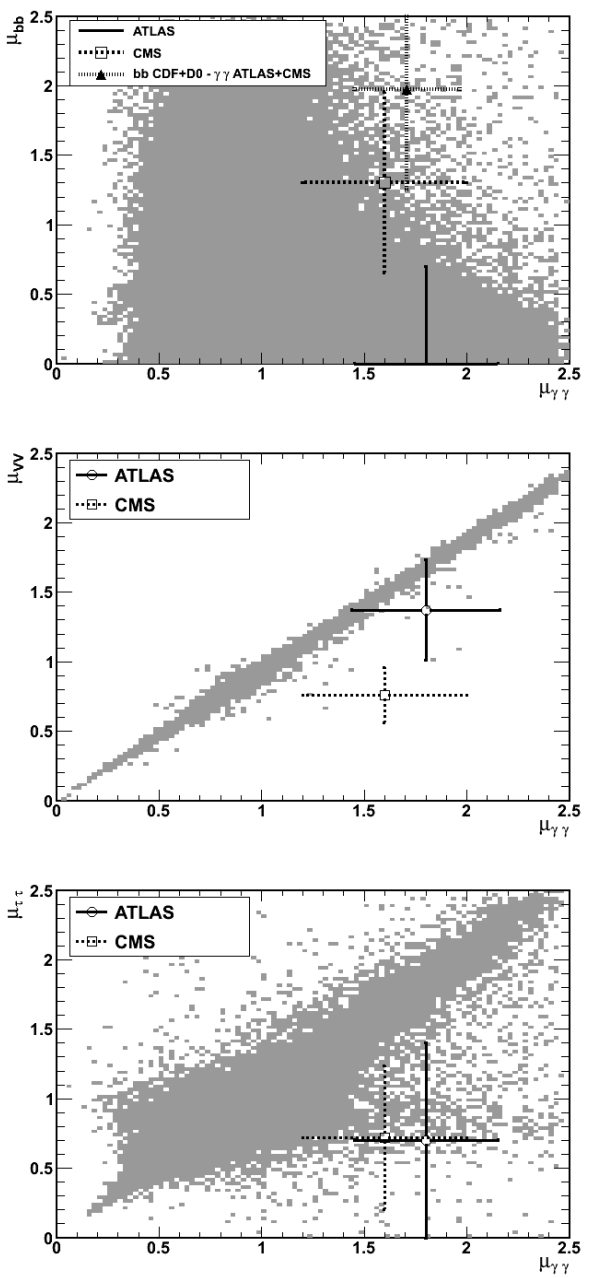

Figure 1: The $b \bar{b}$ (top), $V V=W W+Z Z$ (centre) and $\tau \tau$ (bottom) signal strengths vs. that for $\gamma \gamma$ from the LHC and Tevatron results compared to the values for the accepted pMSSM points.

Then, we move to consider the compatibility of the $h$ mass and signal rates predicted for the accepted pMSSM points with the updated LHC measurements. The results on the signal strengths from the current results for the individual experiments are compared to the distributions obtained for accepted pMSSM points in Figure 1. We use the signal strengths for the channels where a signal has been observed, $\mu_{\gamma \gamma}, \mu_{Z Z}, \mu_{W W}$ and we also add the limits obtained for $\mu_{b \bar{b}}$ and $\mu_{\tau \tau}$. The systematic uncertainties from the 
Higgs production cross section in the $g g \rightarrow h$ channels may be sizeable [22], at least $\pm 15 \%$, and have been taken into account. The use of ratios of the signal strengths reduces these uncertainties and we thus also test the signal strength ratios $D_{\gamma \gamma}=\mu_{\gamma \gamma} / \mu_{V V}$ and $D_{\tau \tau}=\mu_{\tau \tau} / \mu_{V V}$ [23], where $\mu_{V V}$ is the weighted average of the signal strengths in the $W W$ and $Z Z$ channels, which are mostly immune from these systematics

In order to evaluate the compatibility of each point with the Higgs results, we compute the total $\chi^{2}$ probability for the observables of Table 1 for each accepted pMSSM point. The $\chi^{2}$ for a given pMSSM point is built as

$$
\chi^{2}=\frac{\left(M_{h}(L H C)-M_{h}(i)\right)^{2}}{\delta^{2}\left[M_{h}(L H C)\right]+\delta^{2}\left[M_{h}(t h)\right]}+\sum_{j} \frac{\left(\mu_{j}(L H C)-\mu_{j}(i)\right)^{2}}{\delta^{2}\left[\mu_{j}(L H C)\right]+\delta^{2}\left[\mu_{j}(t h)\right]}
$$

where $i$ is the index of the pMSSM point, $M_{h}(L H C) \pm \delta\left[M_{h}(L H C)\right]$ and $\mu_{j}(L H C) \pm \delta\left[\mu_{j}(L H C)\right]$ the LHC (and Tevatron) measurements of the mass and the signal strengths in channel $j$ with their uncertainties as given in Table 1 and the theory uncertainties $\delta^{2}\left[M_{h}(t h)\right]$ and $\delta^{2}\left[\mu_{j}(t h)\right]$ account for the theory systematics on the MSSM $h$ mass, $\pm 1.5 \mathrm{GeV}$ and the production rate.

For the $b \bar{b}$ and $\tau^{+} \tau^{-}$channels, where no signal evidence has been reported, we add the contribution to the total $\chi^{2}$ only when the respective $\mu$ value is outside the $\pm 1.5 \sigma$ interval from the measured central value, and the pMSSM point becomes increasingly less consistent with the limits reported by the LHC and Tevatron experiments.

\subsection{SUSY corrections to the Higgs rates}

In general, deviations of the $\mu$ signal strength ratios from their SM values may be due to modifications of either the decay branching fractions or the relevant production cross sections, or to both. In order to disentangle these effects, it is important to conduct analyses where the same decay channel is studied in different production processes, such as gluon fusion $g g \rightarrow h$, associated production with a gauge boson (VH) or forward jets (VBF). The ATLAS collaboration published a first attempt to separate the contribution of the VBF and VH production from $g g \rightarrow h$ in the $h \rightarrow \gamma \gamma$ channel [1]. The confidence level (C.L.) contours obtained in the analysis are compared in Figure 2 to the distribution for all the accepted pMSSM points and to those selected within the $90 \%$ C.L. with the Higgs results from the $\chi^{2}$ probability analysis. 


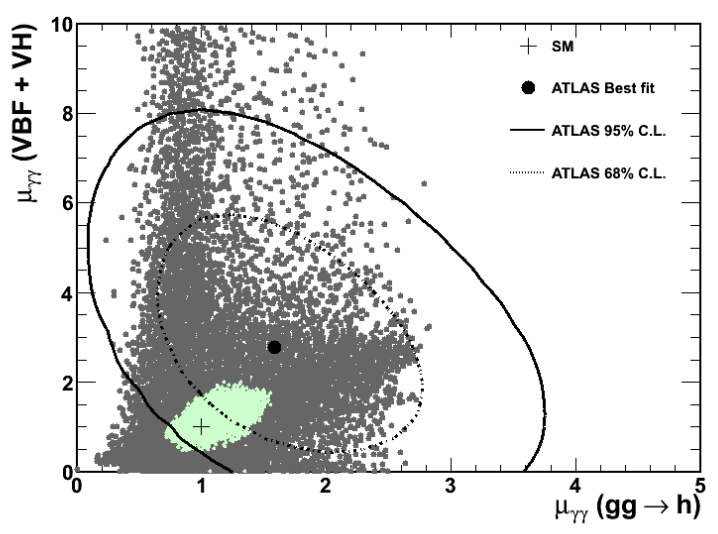

Figure 2: $\mu$ values in the $h \rightarrow \gamma \gamma$ channel for associate VBF and VH production vs. $g g \rightarrow h$. The dots in dark grey show the accepted pMSSM points and those in light green the points which agree at 90\% C.L. with the constraints of Table 1. The contours give the results obtained by the ATLAS experiment (adapted from Ref. [1]).

The $h$ decay branching fractions may be modified by a change of the $h$ total decay width. Since the dominant decay mode for a $\sim 126 \mathrm{GeV}$ lightest $h$ boson is $h \rightarrow b \bar{b}$, a change of the effective $h b \bar{b}$ coupling by direct vertex corrections, through the $\Delta_{b}$ correction that grows as $\mu \tan \beta$, results in an anti-correlated variation of the branching fractions of all the other modes compared to that in $b \bar{b}$. The reduction of the $h \rightarrow b \bar{b}$ decay width, away from the decoupling regime $M_{A} \gg M_{Z}$, occurs in a non-trivial way. The radiative corrections to the mixing angle $\alpha$ in the $\mathrm{CP}$-even Higgs sector strongly affect the $h b \bar{b}$ coupling, $g_{h b \bar{b}}=-\sin \alpha_{\text {eff }} / \cos \beta$. While in the decoupling limit we expect $\tan \alpha_{\text {eff }} \rightarrow-1 / \tan \beta$ making $g_{h b \bar{b}}$ to become SM-like, there is a combination of parameters which realises the so-called "vanishing coupling" regime [24] in which $\alpha_{\text {eff }} \rightarrow 0$. In this case, $\left(\tan \alpha_{\text {eff }} \tan \beta\right)$ becomes very small and when $\mu$ is positive, we obtain an additional reduction of the $h b \bar{b}$ coupling by a factor $\approx 1-\Delta_{b} /\left(\tan \alpha_{\text {eff }} \tan \beta\right)$. This combination of parameters leads to a reduction of the decay rate 11 for $h \rightarrow b \bar{b}$ thereby enhancing all other channels, including $h \rightarrow \gamma \gamma$. This would explain a possible excess in the rate

\footnotetext{
${ }^{1}$ Note that in this small $\alpha_{\text {eff }}$ scenario, the rate for the $h \rightarrow \tau^{+} \tau^{-}$channel will also be suppressed since $g_{h \tau \tau} \propto-\sin \alpha_{\text {eff }} / \cos \beta$. In turn, there is no significant change by $\Delta_{\tau}$ corrections, that are similar to $\Delta_{b}$ for the electro-weak part but much smaller (they are now included in the program HDECAY 5.0 [21]).
} 
of the $\gamma \gamma$ channel without any modification to the $g g \rightarrow h$ production rate or the $h \rightarrow \gamma \gamma$ branching fraction. In turn, this effect should have no impact on the ratio of decay widths, $D_{\gamma \gamma}$, which does not depend on the total Higgs width. The dependence of $R_{b b}$ on $\mu \tan \beta$ through the $\Delta_{b}$ correction and on $\sin \alpha_{\text {eff }}$, i.e. on the $h b \bar{b}$ coupling without the vertex corrections, are shown in Figure 3. For small values of $\sin \alpha_{\text {eff }}$ the variations of $R_{b b}$ from the $\Delta_{b}$ term are enhanced, increasing or decreasing its value depending on the sign of $\mu$.
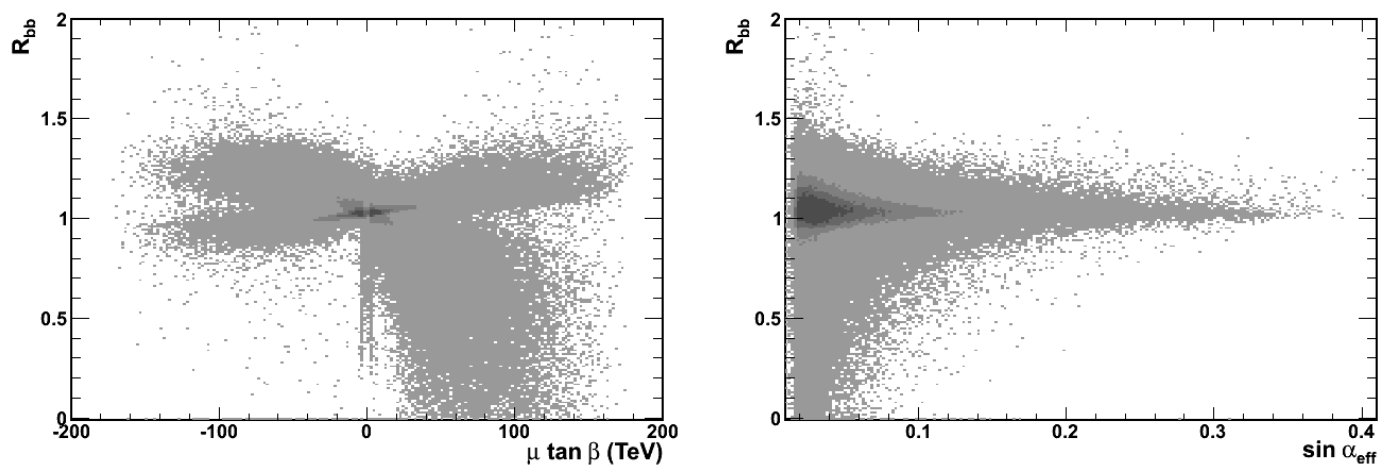

Figure 3: Dependence of the $h \rightarrow b \bar{b}$ branching fraction normalised to the SM expectation as a function of $\mu \tan \beta$ (left) and $\sin \alpha_{\text {eff }}$ (right). The intensity of the grey tones is proportional to the density of pMSSM points, which are peaked around $R_{b b} \sim 1$

The total width can also be modified by additional decay channels to SUSY particles. Because of the LEP2 constraints, the only possible channel for the $h$ boson is the invisible decay into pairs of the lightest neutralinos $h \rightarrow \chi_{1}^{0} \chi_{1}^{0}$. The invisible width can be important for $M_{\tilde{\chi}_{1}^{0}}<60 \mathrm{GeV}$ and for not too large $M_{1}$ and $|\mu|$ values, and may substantially suppress the decays into SM particles. This potentially large effect can be revealed by a combined study of the individual signal strength values in the various visible Higgs decay channels, since the changes in these channels are correlated, but not from the ratio $D_{\gamma \gamma}$. Upper bounds on the invisible decay rate have been obtained from the measured signal strengths [25, 26].

The neutralino LSP, with such small mass, would have the relic density required by the WMAP results, since it will annihilate efficiently through the exchange of the $h$ boson. However, in this case the invisible branching fraction should be small. This is exemplified in Figure 4 where $\log _{10}\left(\Omega_{\chi} h^{2}\right)$ is shown as a function of $M_{\chi_{1}^{0}}$ for the accepted set of pMSSM points and for those which have $\operatorname{BR}\left(h \rightarrow \chi_{1}^{0} \chi_{1}^{0}\right) \geq 15 \%$, close to the $68 \%$ C.L. upper limit 

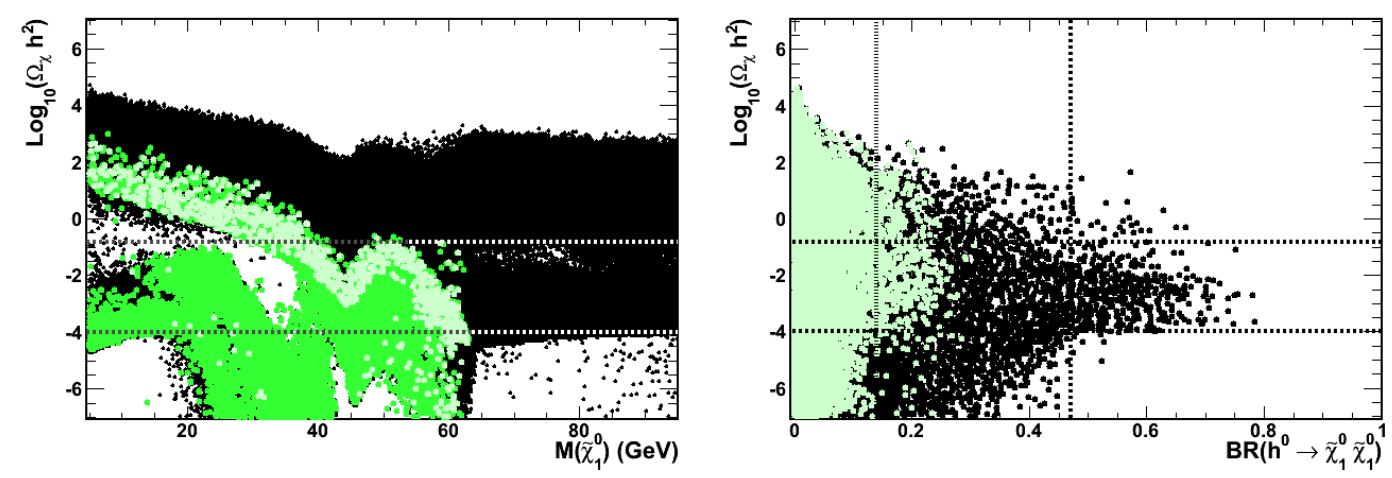

Figure 4: The neutralino relic density $\log _{10}\left(\Omega_{\chi} h^{2}\right)$ as a function of $M_{\chi_{1}^{0}}$ (left) and $\mathrm{BR}\left(h \rightarrow \chi_{1}^{0} \chi_{1}^{0}\right)$ (right) for the accepted set of pMSSM points (black dots), those with $\operatorname{BR}\left(h \rightarrow \chi_{1}^{0} \chi_{1}^{0}\right) \geq 15 \%$ (green dots) and those compatible at $90 \%$ C.L. with the Higgs data (light green dots). The horizontal lines show the constraint imposed on $\Omega_{\chi} h^{2}$ and the vertical lines on the panel on the right the $68 \%$ and $95 \%$ C.L. constraints on the Higgs invisible decay branching fraction obtained by [26].

obtained in [26]. As can be seen only a small area in the region $30 \lesssim M_{\chi_{1}^{0}} \lesssim$ $60 \mathrm{GeV}$ fulfils this last condition and the $\Omega_{\chi} h^{2}$ constraint.

We consider now the contributions of SUSY particles to the $\gamma \gamma$ branching fraction and, eventually, to the $g g \rightarrow h$ amplitude [27, 28]. Even though the individual contributions give corrections of $\mathcal{O}(10 \%)$ and in some cases more, it is interesting to observe that different corrections can sum up, resulting in sizeable overall shifts of the branching fractions compared to their SM values. These contributions come from light scalar top and bottom quarks, staus and charginos, as briefly summarised below.

a) Stop squark loops: as already discussed in [16, 15], the Higgs mass constraint requires a very large SUSY scale $M_{S}=\sqrt{m_{\tilde{t}_{1}} m_{\tilde{t}_{2}}}$ and/or a large value of the stop mixing parameter $X_{t}=A_{t}-\mu / \tan \beta$ to maximise the radiative corrections to $M_{h}$. If $\tilde{t}_{1}$ is light, $M_{\tilde{t}_{1}} \lesssim 500 \mathrm{GeV}$, the mixing term must be $X_{t} \approx \sqrt{6} M_{S}$ to obtain $M_{h} \approx 126 \mathrm{GeV}$. In this case the $h \tilde{t}_{1} \tilde{t}_{1}$ coupling, that is also proportional to $X_{t}$, becomes large and leads to sizeable stop loop contributions to the induced Higgs couplings to gluons and photons. However, a $h \rightarrow \gamma \gamma$ rate enhancement is compensated by a suppression of the $g g \rightarrow h$ production cross section.

b) Light sbottom squarks: a light right-handed $\tilde{b}_{R}$ state, as $\tilde{b}_{L}$ which belongs to the same iso-doublet as $\tilde{t}_{L}$ should be heavier, does not conflict with the $M_{h}$ value since the radiative corrections from the sbottom sector 
are in general small. For $M_{\tilde{b}_{1}} \lesssim 400 \mathrm{GeV}$, it contributes to the $h g g$ vertex and slightly enhances the $g g \rightarrow h$ production rate. In turn, it would have little impact on the $h \rightarrow \gamma \gamma$ rate because of the largely dominating $W$ loop and the small $\tilde{b}_{1}$ electric charge. Hence, the $g g \rightarrow h \rightarrow \gamma \gamma$ rate could be slightly enhanced by light sbottoms.

c) Light $\tilde{\tau}$ sleptons: they have received most of the attention in the literature as it might lead to the largest contributions, see e.g. Refs. [28]. For low stau mass parameters $M_{\tilde{\tau}_{L}}, M_{\tilde{\tau}_{R}} \approx$ a few $100 \mathrm{GeV}$, and large stau mixing parameter $X_{\tau}=A_{\tau}-\mu \tan \beta$, with $\tan \beta \approx 60$ and $|\mu|=500-1000 \mathrm{GeV}$ leading to $\left|X_{\tau}\right| \approx 30-60 \mathrm{TeV}$, the lighter $\tilde{\tau}_{1}$ state has a mass close to the LEP2 bound, $M_{\tilde{\tau}_{1}} \approx 100 \mathrm{GeV}$ and its coupling to the $h$ boson, $g_{h \tilde{\tau} \tilde{\tau}} \propto M_{\tau} X_{\tau}$, is large. The $\tilde{\tau}_{1}$ contribution, proportional to $M_{\tau}^{2} X_{\tau}^{2} / M_{\tilde{\tau}_{1}}^{2} M_{\tilde{\tau}_{2}}^{2}$, can be large enough to significantly increase $\operatorname{BR}(h \rightarrow \gamma \gamma)$ [28] with a change of up to $50 \%$, for extreme choices of the parameters.

d) Chargino loops: the Higgs couplings to charginos are very small if these are pure winos or higgsinos, and maximal for states with equal higgsinowino mixture. Contrary to the scalar case, where the loop contributions are damped by $1 / \tilde{M}^{2}$, the chargino contributions to the $h \rightarrow \gamma \gamma$ amplitude are damped only by $1 / M_{\tilde{\chi}_{i}^{ \pm}}$factors, so that the decoupling of the charginos from the vertex occurs more slowly. However, for a chargino mass $M_{\tilde{\chi}_{1}^{ \pm}} \gtrsim 100 \mathrm{GeV}$ and maximal couplings to the $h$ boson, the corrections to the $h \rightarrow \gamma \gamma$ rate do not exceed the 10-15\% level. The sign of the correction depends on the sign of $\mu$, with the enhancement occurring for $\mu>0$, as for the $\Delta_{b}$ correction.

\subsection{Constraints on MSSM parameters}

In order to study the constraints on the MSSM parameters deriving from the Higgs data, we compute the $\chi^{2}$ probability for the accepted points with $M_{h}>114 \mathrm{GeV}$ and select those compatible at $90 \%$ and $68 \%$ C.L. with the Higgs constraints of Table 1. Results are summarised in Figure 5, where we show the accepted pMSSM points with $M_{h}>114 \mathrm{GeV}$ and those compatible with the observed $h$ mass and signal strengths at the $90 \%$ and $68 \%$ C.L. in the $\left[M_{A}, \tan \beta\right],\left[M_{\tilde{t}_{1}}, X_{t}\right],\left[M_{\tilde{b}_{1}}, X_{b}\right],\left[M_{\tilde{\tau}_{1}}, X_{\tau}\right],\left[\mu, M_{1,2}\right]$ planes.

Given the present statistical accuracy of the LHC results, the $90 \%$ C.L. regions, which contain $28 \%$ of the accepted points, have little discriminant power since all the measured signal strengths agree with the SM expectations at this confidence level. On the contrary, the $68 \%$ C.L. regions, containing $7.7 \%$ of the accepted points, clearly highlight specific regions in the chosen 

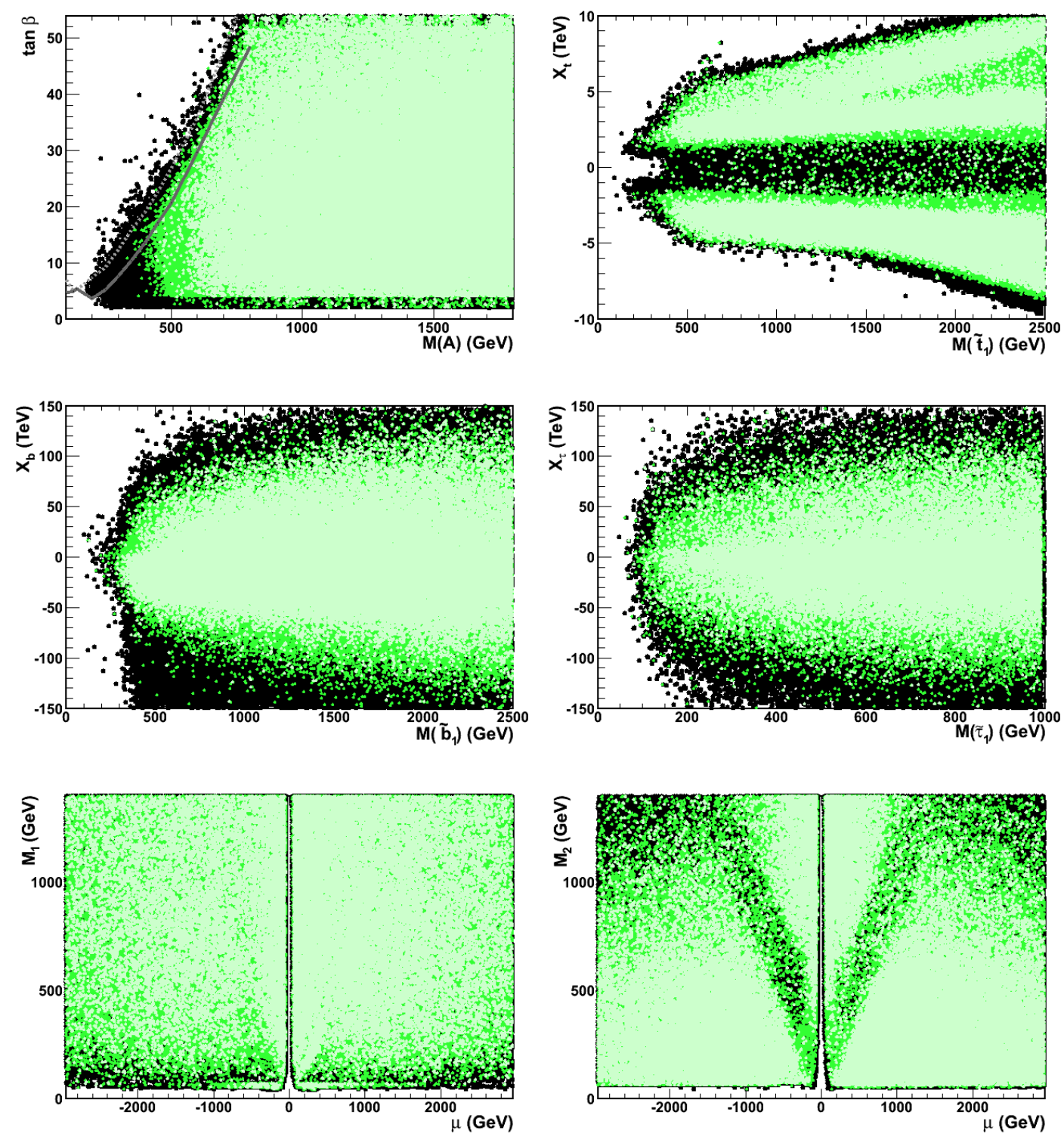

Figure 5: Distributions of the pMSSM points in various pMSSM planes. The black dots show the accepted pMSSM points with $M_{h}>114 \mathrm{GeV}$, those in dark (light) green the points compatible with the mass and rate constraints of Table 1 at $90 \%$ $(68 \%)$ C.L. On the $\left[M_{A}, \tan \beta\right]$ histogram (upper left), the $95 \%$ C.L. expected (dotted line) and observed (continuous line) limit from the $H / A \rightarrow \tau \tau$ search of Ref. [12] are superimposed. 
parameter sets, where the discrimination is driven mostly by $M_{h}$ and the interplay of the $\mu_{\gamma \gamma}$ value and the $\mu_{b b}$ and $\mu_{\tau \tau}$ limits.

While the $\mu_{X X}$ values are sensitive to corrections to both the Higgs width and the loop effects to the $h X X$ couplings, the $D_{X X}$ values are only sensitive to the latter, since the width effect gets cancelled in the ratio. The current LHC accuracy does not yet provide sensitivity to the bulk of these loop effects, expected to be at the $\mathcal{O}(10 \%)$ level. Using the $D_{\gamma \gamma}$ ratio as a constraint at the $90 \%$ C.L, only $0.4 \%$ of the accepted points are kept. These consists mostly of to points with the $\tilde{\tau}_{1}$ mass in the range between the LEP2 limit and $\sim 200 \mathrm{GeV}$ and intermediate values of $X_{\tau}$ (see Figure 6), correspond to the scenario c) discussed above in Section 2.1, where the $\gamma \gamma$ rate is enhanced by light $\tilde{\tau}$ loops. Finally, we compare the fraction of accepted

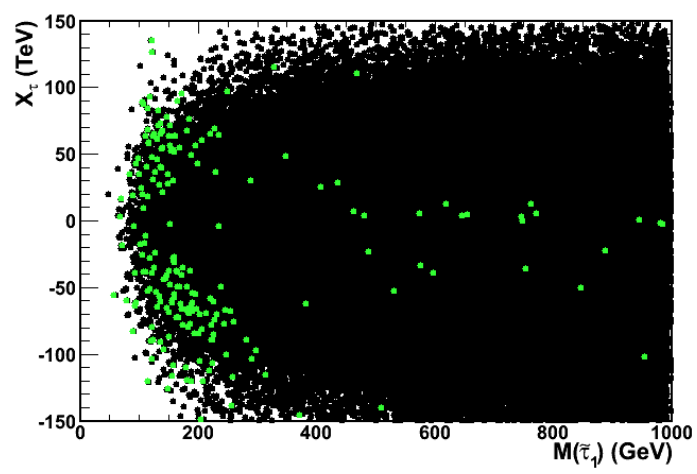

Figure 6: Distributions of the pMSSM points in the $\left[M_{\tilde{\tau}_{1}}, X_{\tau}\right]$ plane. The black dots show the accepted pMSSM points with $M_{h}>114 \mathrm{GeV}$, those in dark green the points compatible with the $D_{\gamma \gamma}$ constraint of Table 1 at $90 \%$ C.L.

pMSSM points, with $M_{h}>114 \mathrm{GeV}$, compatible at $68 \%$ C.L. for the full set of observables, which is $7.7 \%$ when we consider the theory systematics and becomes $0.2 \%$ and $0.1 \%$ for the $M_{h}, \mu_{\gamma \gamma}, \mu_{Z Z}, \mu_{W W}$ set of observables and the full set of observables, without accounting for the production cross section uncertainties, respectively.

As can be observed from the $\left[M_{A}, \tan \beta\right]$ plot, the data prefer the decoupling regime with $M_{A} \gtrsim 400 \mathrm{GeV}$ for all $\tan \beta$ values and even higher $M_{A}$ at large $\tan \beta$. There are however some exceptions and a few points still survive the strong CMS limit from the $H / A \rightarrow \tau^{+} \tau^{-}$negative search as will be discussed in more detail shortly.

As already discussed in Refs. [16, 15] and elsewhere, the lighter stop 
state can still have a mass of about $500 \mathrm{GeV}$, but a strong stop mixing, $X_{t} \approx \sqrt{6} M_{S}$, is then needed in order to accommodate the $M_{h}=126 \pm 3$ $\mathrm{GeV}$ value. Positive values of $X_{t} \approx A_{t}$ are slightly favoured as they allow a better maximisation of the $M_{h}$ value. In the sbottom case, the region with small $M_{\tilde{b}_{1}}$ and moderate mixing is favoured as it leads to light sbottoms that would slightly enhance the $g g \rightarrow h \rightarrow \gamma \gamma$ rate. For increasing $M_{\tilde{b}_{1}}$ values, the mixing parameter $X_{b} \approx-\mu \tan \beta$ tends to be larger which increases the $\Delta_{b}$ corrections and, hence, changes the rate of $R_{b b}$ in the regime where the $h b \bar{b}$ coupling is not SM-like as discussed previously.

In the $\left[M_{\tilde{\tau}_{1}}, X_{\tau}\right]$ plane, a region preferred by the data is the small area with $M_{\tilde{\tau}_{1}}=100-200 \mathrm{GeV}$, which results in an enhancement of the $h \rightarrow \gamma \gamma$ rate. At large values of $M_{\tilde{\tau}_{1}}$ for which the stau does not contribute anymore to the $h \gamma \gamma$ vertex, there is still a preference for large $X_{\tau}$ values but this is mainly due to the fact that, at large $\tan \beta, X_{\tau} \approx X_{b} \approx-\mu \tan \beta$ and, thus, the rate $R_{b b}$ is again affected.

In the $\left[M_{1}, \mu\right]$ and $\left[M_{2}, \mu\right]$ planes, the trend is again mainly driven by the $\Delta_{b}$ correction, as the electro-weak SUSY corrections to this quantity involve several different terms: a term $\propto A_{t} \mu \tan \beta$ from stop contributions, and terms $\propto M_{2} \mu \tan \beta$ and $\propto M_{1} \mu \tan \beta$ from the wino and the bino contributions. An exception is for a very small area with $|\mu| \approx M_{2} \approx 100 \mathrm{GeV}$ where charginos contribute directly to the $h \gamma \gamma$ vertex.

Hence, sbottom mixing plays a major role in this analysis as it affects strongly the $h \rightarrow b \bar{b}$ decay rate and hence the branching fractions for all other decay channels. This is the reason why the behaviour is rather different from what was observed with the summer data with $\approx 10 \mathrm{fb}^{-1}$, since, the first result on the signal strength in the $h \rightarrow b \bar{b}$ channel was below unity (and in Ref. [16] the CDF/D0 data were not included).

\subsubsection{Identification of the observed Higgs state in MSSM}

It has been advocated that the observed $126 \mathrm{GeV}$ particle could indeed be the heavier $H$ boson [29]. This may occur at low values of $M_{A}(\approx 100-120$ $\mathrm{GeV})$, and moderate values of $\tan \beta(\approx 10)$. In this scenario, the $H$ particle has approximately SM-like properties, while the $h$ boson has suppressed couplings to vector bosons and a mass of order $100 \mathrm{GeV}$ or below. In Ref. [15], we performed a dedicated scan for this region of parameter space and found that only $\approx 2 \times 10^{-5}$ of the generated points would remain after imposing the LHC data constraints. These points were then excluded by applying the constraints from flavour physics. The most efficient constraint to this 
scenario were the $A / H \rightarrow \tau^{+} \tau^{-}$limits obtained by the ATLAS and CMS collaborations. This search has been updated by the CMS collaboration based on $12 \mathrm{fb}^{-1}$ of $8 \mathrm{TeV}$ data and the results exclude values of $\tan \beta \gtrsim 5$ in the entire mass range $90<M_{A}<250 \mathrm{GeV}$.

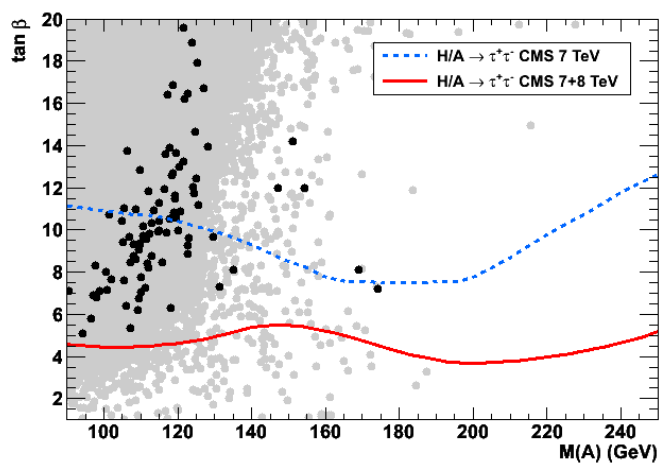

Figure 7: The parameter space $\left[M_{A}, \tan \beta\right]$ with points for the heavier $H$ boson to be observed with a mass in the interval 123-129 GeV (light grey points) and after flavour and dark matter relic density $10^{-4}<\Omega h^{2}<0.155$ constraints (black points). None of these points have rates compatible with those of Table 1. The CMS excluded regions from the 2011 and $2012 H / A \rightarrow \tau^{+} \tau^{-}$searches are shown by the dashed blue and continuous red lines, respectively.

This new result excludes this scenario as is shown in Figure 7 where we zoom in the $\left[M_{A}, \tan \beta\right]$ plane for low values of the input parameters ${ }^{2}$. The small region in which the $H$ boson was allowed to be the observed state (green points) by the previous $H / A \rightarrow \tau^{+} \tau^{-}$CMS search (dashed blue line), is excluded by the new data. In quantitative terms, we observe no point in this scenario to comply with the flavour, dark matter and 90\% C.L. for the Higgs data, which corresponds to a probability of less than $3 \times 10^{-8}$ for our scan points to realise this scenario even before imposing the latest CMS $\tau \tau$ search limits. Conversely, lifting the Higgs rate constraints and imposing the $\tau \tau$ limit leaves us with no viable point for this scenario.

We also note that these new limits also exclude the so-called "intense coupling regime" [32, where the three neutral Higgs bosons could be light and close in mass (in Ref. [15], a very small area of the parameter space at

\footnotetext{
${ }^{2}$ In Ref. [15, a few points allowed in this scenario were ruled out by the $b \rightarrow s \gamma$ constraint. In addition, these points did not satisfy the WMAP constraint of $10^{-4}<$ $\Omega h^{2}<0.155$ [30] when accounting for theoretical and cosmological uncertainties [31.
} 
$M_{A} \approx 140 \mathrm{GeV}$ and $\tan \beta \approx 8$ was still left out).

\subsection{2. $H / A$ decays into SUSY particles}

There is however a caveat to these $H / A \rightarrow \tau^{+} \tau^{-}$constraints. First, large $\Delta_{b}$ corrections could significantly enhance the $H / A \rightarrow b \bar{b}$ decay widths and hence suppress the branching ratio $\mathrm{BR}\left(H / A \rightarrow \tau^{+} \tau^{-}\right)$to make the LHC constraint less efficient. Some (not too large) values of $\tan \beta$ that are presently excluded could be then resurrected. However, this can occur only for very large $\Delta_{b}$ values, $\mathcal{O}(1)$, and hence extreme choice of the pMSSM parameters. Another possibility leading to the suppression of the $H / A \rightarrow$
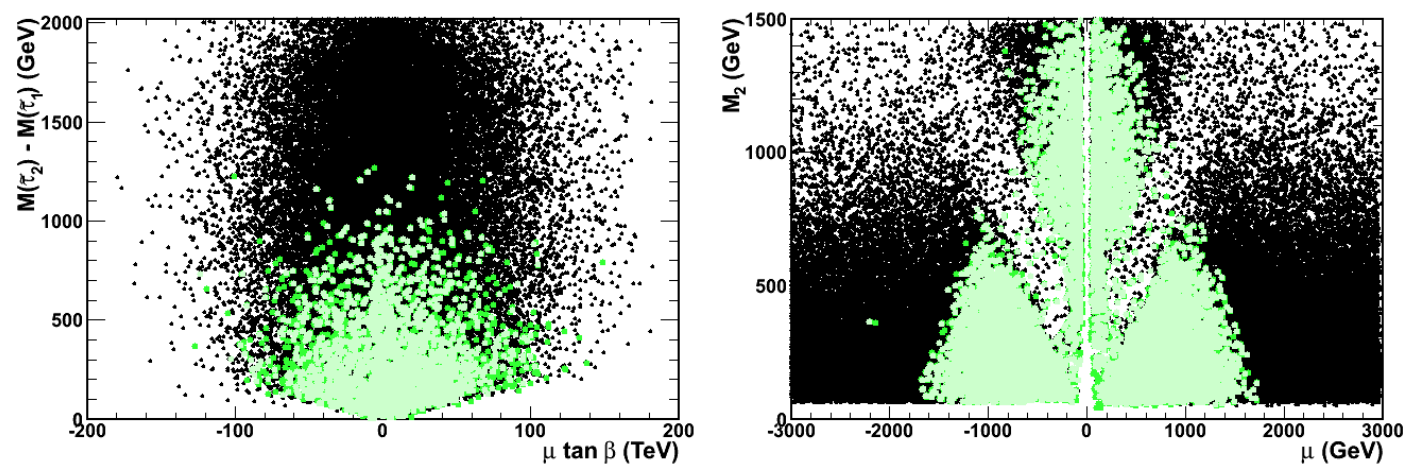

Figure 8: Decays of the $H$ boson into SUSY particles. The left panel shows the allowed region for the $H \rightarrow \tilde{\tau}_{1} \tilde{\tau}_{1,2}$ decay. The black points indicate the area in which the decay is kinematically possible, the dark green those with branching ratios larger than $15 \%$ and the light green the subset fulfilling also the constraints of Table 1 at $90 \%$ C.L. The right panel shows the same for the $H \rightarrow \chi \chi$ where $\chi \chi$ indicates any pair of charginos or neutralinos. The black points indicate the accepted pMSSM points, the dark green those with branching ratios larger than $15 \%$ and the light green the subset fulfilling also the constraints of Table 1 at $90 \%$ C.L

$\tau^{+} \tau^{-}$rate is when the decay channels into SUSY particles are kinematically accessible. This is particularly important in the case of the decays $H \rightarrow \tilde{\tau}_{1} \tilde{\tau}_{1}$ and $H / A \rightarrow \tilde{\tau}_{1} \tilde{\tau}_{2}$ (because of $\mathrm{CP}$ invariance the $A$ boson cannot decay into two sfermions of the same nature) and to a lesser extent $H / A \rightarrow \chi_{i}^{0} \chi_{j}^{0}$ and $\chi_{i}^{+} \chi_{j}^{-}$which can be significant for not too large values of $\tan \beta$ for which the total $H / A$ widths are not too strongly enhanced. This is shown in Figure 8 where the points having a branching fraction $\operatorname{BR}\left(H \rightarrow \tilde{\tau}_{1} \tilde{\tau}_{1,2}\right)$ larger than $15 \%$ are displayed in the plane $\left[M_{\tilde{\tau}_{2}}-M_{\tilde{\tau}_{1}}, \mu \tan \beta\right]$. This is typically the 
area in which we have light staus with large couplings to the Higgs, yielding also an enhancement of $h \rightarrow \gamma \gamma$. The branching fractions into charginos and neutralinos are less significant even for $\tan \beta \lesssim 10$. The invisible decays $H / A \rightarrow \chi_{1}^{0} \chi_{1}^{0}$ have more phase space, but the rate is generally small as the LSP has to be bino-like if is light enough and it thus couples only weakly to the Higgs bosons (Figure 8).

\subsubsection{Constraints for DM direct detection}

Finally, we should note that the Higgs data have also an impact on direct dark matter searches, which are now starting to probe the bulk of the region of the neutralino-nucleon scattering cross section predicted by the MSSM. In particular, the latest results reported by the XENON collaboration improved the earlier $95 \%$ C.L. limit by a factor of $\approx 4$.

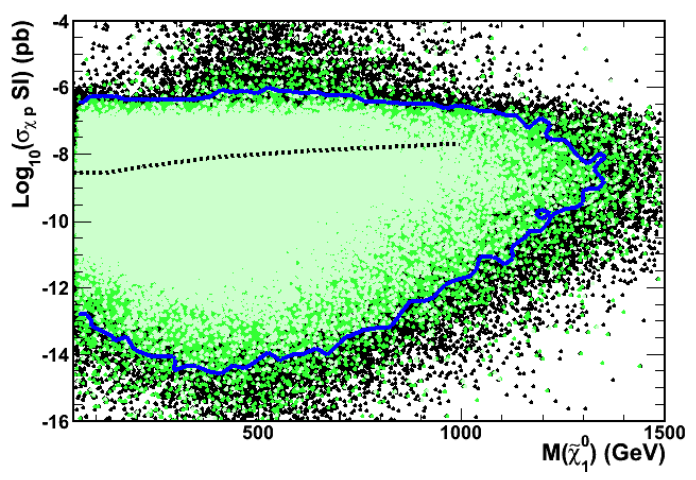

Figure 9: $\chi-p$ scattering cross section as a function of the $\chi_{1}^{0}$ mass. The black dots represent valid pMSSM points, the dark grey dots the subset of points compatible at $90 \%$ C.L. with the LHC Higgs results and the light grey dots compatible at $68 \%$ C.L. The region enclosed by the grey continuous line contains $99.5 \%$ of the points compatible at $90 \%$ C.L. with the LHC Higgs results. The dashed line represents the $95 \%$ C.L. upper limit contour set by the XENON100 experiment with 225 live days of data.

We compare the new XENON limit with the predicted spin-independent $\chi-p$ cross sections as a function of the LSP mass for the points fulfilling various selections in Figure 9. The XENON limit removes $28 \%$ of the accepted MSSM points before the constraints from the LHC Higgs results are applied. This fraction decreases to $24 \%$ and $15 \%$ when we restrict to the points compatible with the measured Higgs mass and rates at, respectively, the $90 \%$ and $68 \%$ C.L. This indicates that the pMSSM points favoured by the LHC 
Higgs results, tend to have a lower $\chi-p$ scattering cross section, as a result of the large value of $M_{A}$ that they imply.

\section{Conclusions}

In this paper, we have updated the study of the phenomenological MSSM performed in Ref. 15 by including the new experimental data recently released. We performed a $\chi^{2}$ probability analysis based on a sample of $2.0 \times 10^{8}$ generated pMSSM points and presented the regions of the relevant MSSM parameters which show agreement at the $68 \%$ and $90 \%$ C.L. with the updated LHC results. The new, and more precise, ATLAS and CMS data for the decay channels $h \rightarrow \gamma \gamma, W^{+} W^{-}, Z Z, b \bar{b}$ and $\tau^{+} \tau^{-}$, the updated CMS constraints from the $H / A \rightarrow \tau^{+} \tau^{-}$search mode, and the new LHCb result for the $B_{s}^{0} \rightarrow \mu^{+} \mu^{-}$decay branching fraction have a significant impact on the pMSSM parameter space.

Our earlier results stay qualitatively the same and are even strengthened. In particular, we have shown that the possibility of being outside the decoupling regime for the MSSM Higgs sector by, for instance, allowing the heavier $\mathrm{CP}$-even $H$ state to be the observed Higgs particle, is now being ruled out. The scenario in which the total $h$ boson decay width is suppressed, in particular when the $h b \bar{b}$ coupling is not SM-like even for $M_{A} \gg M_{Z}$, which enhances the branching fractions for some of the channels still plays a role in view of the increased statistical significance of a possible enhancement in the rate of the $h \rightarrow \gamma \gamma$ decay channel. This also suggests the possibility of some light supersymmetric particles, such as staus, charginos and third generation squarks, contributing to the $h \gamma \gamma$ loop-induced vertex. Nevertheless, for not too extreme choices of the pMSSM parameters, we find that the contributions of the SUSY particles to the $h \rightarrow \gamma \gamma$ branching fraction should not exceed, in general, the $\approx 20 \%$ level.

\section{Acknowledgements}

We thank N. Berger and M. Spira for discussion, M. Schumann and P. Beltrame for providing the XENON results in numerical form. AD thanks the CERN TH unit for hospitality.

\section{References}

[1] G. Aad et al. [ATLAS collaboration], Phys. Lett. B716 (2012) 1. 
[2] S. Chatrchyan et al. [CMS collaboration], Phys. Lett. B716 (2012) 30.

[3] [ATLAS Collaboration], Note ATLAS-CONF-2012-168.

[4] [ATLAS Collaboration], Note ATLAS-CONF-2012-169.

[5] [CMS Collaboration], Note CMS PAS HIG-2012-015.

[6] [CMS Collaboration], Note CMS PAS HIG-2012-016.

[7] [ATLAS Collaboration], Note ATLAS-CONF-2012-158.

[8] [ATLAS Collaboration], Note ATLAS-CONF-2012-161.

[9] [CMS Collaboration], Note CMS PAS HIG-2012-044.

[10] [ATLAS Collaboration], Note ATLAS-CONF-2012-160.

[11] [CMS Collaboration], Note CMS PAS HIG-2012-043.

[12] [CMS Collaboration], Note CMS PAS HIG-2012-050.

[13] R. Aaij et al. [LHCb Collaboration], CERN-PH-EP-2012-335.

[14] E. Aprile et al. [XENON100 Collaboration], Phys. Rev. Lett. 109 (2012) 181301.

[15] A. Arbey, M. Battaglia, A. Djouadi and F. Mahmoudi, JHEP 1209 (2012) 107.

[16] A. Arbey et al., Phys. Lett. B708 (2012) 162.

[17] A. Arbey, M. Battaglia and F. Mahmoudi, Eur. Phys. J. C72 (2012) 1847; Eadem, Eur. Phys. J. C72 (2012) 1906.

[18] The CDF and D0 Collaborations, FERMILAB-CONF-12-318-E.

[19] F. Mahmoudi, S. Neshatpour and J. Orloff, JHEP 1208 (2012) 092.

[20] Y. Amhis et al., (HFAG collaboration), arXiv:1207.1158[hep-ex].

[21] A. Djouadi, J. Kalinowski and M. Spira, Comput. Phys. Commun. 108 (1998) 56. 
[22] S. Dittmaier et al., [LHC Higgs cross section working group], arXiv:1101.0593 [hep-ph]; J. Baglio and A. Djouadi, JHEP 1103 (2011) 055 .

[23] A. Djouadi, arXiv:1208.3436 [hep-ph].

[24] M. Carena, S. Heinemeyer, C. Wagner and G. Weiglein, Eur. Phys. J. C26 (2003) 601.

[25] J. R. Espinosa, M. Muhlleitner, C. Grojean and M. Trott, JHEP 1209 (2012) 126

[26] B. A. Dobrescu and J. D. Lykken, arXiv:1210.3342 [hep-ph].

[27] A. Djouadi, V. Driesen, W. Hollik and J. I. Illana, Eur. Phys. J. C1 (1998) 149; A. Djouadi, Phys. Lett. B435 (1998) 101; A. Djouadi, Phys. Rept. 459 (2008) 1.

[28] See e.g., M. Carena et al., JHEP 1207 (2012) 175; M. Carena, I. Low and C. E. M. Wagner, JHEP 1208 (2012) 060; G. F. Giudice, P. Paradisi, and A. Strumia, JHEP 1210 (2012) 186; U. Haisch and F. Mahmoudi, arXiv:1210.7806 [hep-ph].

[29] See for instance, S. Heinemeyer, O. Stal and G. Weiglein, Phys. Lett. B710 (2012) 201; P. Bechtle et al., arXiv:1211.1955 [hep-ph]; M. Drees, arXiv:1210.6507 [hep-ph].

[30] E. Komatsu et al. [WMAP Collaboration], Astrophys. J. Suppl. 192 (2011) 18.

[31] A. Arbey and F. Mahmoudi, Phys. Lett. B669 (2008) 46; Eadem, JHEP 1005 (2010) 051.

[32] E. Boos et al., Phys. Rev. D66 (2002) 055004; Eadem, Phys. Lett. B578 (2004) 384. 